\title{
LE DISCOURS SUR LE COURAGE ET SUR LA PEUR À L'ÉPOQUE DE LA RENAISSANCE.
}

\author{
JEAN DELUMEAU \\ Collège de France.
}

\section{1. - DES CHEVALIERS TEMERAIRES.}

A l'époque - XVe et XVIe siècles - où l'Occident entre dans les temps modernes le modèle de l'homme courageux le plus couramment reçu reste le chevalier. Dans la droite ligne des épopées, des romans et des chroniques du Moyen Age, toute une littérature écrite, bien sûr, pour la noblesse et les cours - continue d'exalter le preux dont Froissard avait défini les mérites:

"Prouesse, écrivait-il, est si noble vertu et de si grande recommandation qu'on ne doit pas en parler trop brièvement, car elle est mère et lumière des gentilshommes, et, comme la bache ne peut pas brûler sans feu, le gentilhomme ne peut pas accéder à l'honneur parfait, ni à la gloire du monde, sans prouesse" (1).

Trois quarts de siècle plus tard, le même idéal inspire l'auteur de Jehan de Saintré (vers 1456). Pour lui. le chevalier digne de ce titre doit braver tous les dangers par amour de la gloire et de sa dame:

"Le vray amoreux gentilhomme, lit-on dans ce roman, qui n'est point ordonné ne disposé aux estudes de très prudentes et sainctes sciences de théologie, des decrez, des lois ne autres .estudes de sciences, fors que a la tres noble et illustre science et mestier des armes... pour $y$ acquerir honneur et la tres desiree

(1). - FROISSART, Chroniques, éd. S. Luce, Paris, 1869, t. I, p. 2. Cf. notamment G. COHEN, Histoire de la chevalerie en France au Moyen .4ge, Paris, 1949, et M. MOLLAT. Genèse médiévale de la France moderne, Paris, 1970, pp. 210-212. 
grace de sa tresbelle dame..., ce est cellui qui ... fait tant que entre les autres il est nouvelles de lui" (2).

On acquiert d'autant plus d'honneur qu'on risque davantage sa vie dans des combats inégaux. Ceux-ci sont le pain quotidien d'Amadis de Gaule, un héros issu du cycle du roman breton. Repose-toi un peu, lui conseille-t-on au terme d'innombrables aventures,

"Toi qui as vaincu et défait tant de preux chevaliers et tant de géants féroces et redoutables, menant à bien, au très grand péril de ta personne, tous les exploits que tu as entrepris, faisant même trembler les plus cruelles bêtes sauvages par la crainte que leur inspirait la fermeté de ton courage" (3).

Edité en Espagne en 1508, traduit en français à la demande de François 1er, l'Amadis de Gaule et ses suppléments comptent au XVIe siècle plus de soixante éditions espagnoles et une foule de françaises et d'italiennes (4). Exporté en Amérique, il accompagne et inspire les conquistadors - en général des petits nobles - dans leur quête de l'Eldorado. Plus impressionnante encore est la fortune du Roland furieux d'Arioste, peut-être le plus grand succès de librairie du XVIe siècle: quelque 180 éditions de 1516 à 1600 . Roland, "paladin inaccessible à la peur" méprise naturellement "la vile troupe des Sarrasins" qui l'attaquent à Roncevaux. Durandal aidant,

"les bras, les têtes, les épaules (des ennemis) volent de toutes parts; la Mort semble errer parmi les Sarrasins sous mille formes différentes. Elle se dit en elle-même: Durandal dans les mains de Roland vaut mieux encore que cent des faux dont je suis armée" (ch. XII).

Quant aux chevaliers chrétiens que Tasso met en scène dans la Jérusalem délivrée (1ère éd., 1581), arrivant en vue de la ville sainte, ils piaffent d'impatience,

"devancent le signal des trompettes et des tambours, et se mettent en campagne avec de hauts cris d'allégresse".

(2). - A. DE LA SALE, Jehan de Saintré, éd. J. Misrah et Ch. A. Knudson, Genève, 1965, pp. 29-30.

(3). - L. IV, chant L II.

(4). - Les renseignements concernant l'édition au XVIe siècle dans L. FEBVRE et H. J. MARTIN, L'apparition du livre, Paris, 1958, pp. 429-432. 


\begin{abstract}
Godefroy de Bouillon, leur chef, modère difficilement leur ardeur. Car
\end{abstract}

"il est moins aisé de les retenir que de faire aller à contre-mont les flots irrités sur le point de se précipiter dans le gouffre de Charybde, ou d'arrêter les impétueuses haleines de Borée" (ch. III).

$\mathrm{Au}$ modèle correspond une réalité. Des nobles portugais $-\mathrm{Ga}$ ma, Albuquerque, Almeida, Castro etc., - remplissent l'Orient de leurs exploits, fournissant à Camoens la matière d'une épopée moderne. La littérature des chroniques reste, quant à elle, intarissable sur les faits d'armes de la noblesse et des princes, ceux-ci étant la fleur de toute noblesse. Elles les présente comme imperméables à toute crainte. Jean sans peur gagne son nom significatif en 1408 en luttant contre les Liégeois:

"Tant qu'est a parler de la constance, hardiesse et vaillance d'icelui duc de Bourgogne, écrit Enguerrand de Monstrelet, et comment en sa dite bataille, au commencement d'icelle, en décourant de lieu a autre sur un petit cheval, exhorta et bailla a ses gens grand courage, et comment il se maintint jusques en la fin, n'est besoin d'en faire grand declaration; car pour vrai il fit là si grandement qu'il fut loué et prisé de tous ses chevaliers et autres de ses gens..." (5).

Sur Charles le Téméraire - autre surnom à relever - les chroniqueurs bourguignons sont encore plus élogieux.

"Il estoit fier et de haut courage; asseur en péril, sans peur et sans hide (frayeur); et si oncques Hector fut vaillant devant Troyes, cestuy l'estoit autant".

Ainsi parle Chastellain (6). Et Molinet de renchérir après la mort du duc:

"C'estoit ... la plante d'honneur inestimable, l'estoc de grace bieneurée (bienheureuse), et l'arbre de vertu coulourée, redolente (parfumée), fructueuse et de grande altitude".

(5). - Dans Collection des chroniques nationales franf̧aises, éd. J. A. BUCHERON, Paris, 1826 et suiv., pp. 17-18.

(6). - lbid., XLII, p. XXXV. 
D'où les lamentations qu'entraine sa mort tragique:

“... là tresbucha la tres redolente fleur d'honneur es poindans espines; le tres précieux dyamant fut cassé de meschans ferailles et le tres fort et noble lyon aterré de vilaines bestes" (7).

A lire de tels éloges on ne s'étonne pas qu'au siècle suivant François 1er se soit comporté à Pavie avec plus de vaillance que de raison, ni que lui-même et Charles Quint aient échangé des cartels, en 1528, pour un duel judiciaire où ils auraient vidé en combat singulier le contentieux entre Valois et Habsbourg. La société aristocratique de la Renaissance conserve donc l'échelle de valeurs de la période antérieure et, comme elle, cote au plus haut le courage au combat. Aussi bien François 1er tient-il, au soir de Marignan, à se faire armer chevalier par Bayard entré vivant dans la légende parce que "sans paour et sans reproche". La mort du gentilhomme dauphinois en 1524 met "toute noblesse" en "deuil". Car, assure le Loyal Serviteur,

"depuis la création du monde, tant en la loy chrestienne que payenne, ne s'en est trouvé ung seul qui moins luy ai fait de deshonneur ne plus d'honneur ... Il a suivy les guerres ... par l'espace de trente et quatre ans où durant le temps ne s'est trouvé homme qui l'ait passé en toutes choses servans au noble exercice des armes; car de hardiesse peu de gens l'ont approché. De conduyte, c'estoil ung Fabius Maximus; d'entreprises subtiles ung Coriolanus et de force et magnanimité ung second Hector" (8).

Beaucoup de chevaliers qui combattirent à Crécy, à Nicopolis, à Azincourt et à Pavie n'eurent pas la prudence de Fabius ni la "subtilité" de Coriolan. Mais ils s'efforcèrent d'avoir la bravoure d'Hector et ils finirent comme lui. Fr. Guicciardini, relatant la bataille de Pavie, note:

"... on compta parmi les morts vingt des plus grands seigneurs du royaume (de France)". Le Roi vit "tuer ou prendre autour de lui la plupart de ses capitaines et de sa noblesse" (Histoire des guerres d'Italie, 1. XV).

(7). - Ed. G. DOUTREPONT et O. JODOGNE, Bruxelles, 1935-1937, I, p. 207.

(8). - La Tresjoyeuse, plaisante et récréative hystoire du bon chevalier sans paour et sans reproche, composée par le Loyal Serviteur, éd. M. PETITOT, Paris, 1820, Ière série, XVI, 2, pp. 133-134. 
De fait, Bonnivet, La Trémoille. La Palice, parmi beaucoup d'autres, périrent dans la rencontre. Cette tradition de courage militaire resta vigoureuse au cours des âges dans tout un secteur de la noblesse habituée à "jouer avec la mort" (Bernanos) et à risquer sa vie pour le Roi. Fr. Lebrun a suivi, à titre d'échantillon, la destinée d'une famille noble de l'Anjou aux XVIIe et XVIIIe siècles. Jacques de Rougé, premier marquis du Plessis-Bellière, est tué à la bataille de Castellamare en 1654. Son fils Pierre est tué à son tour à la bataille de Saint Gothard en 1664. Son autre fils, François-Henri, meurt en 1692, à Suse au service du Roi. L'aîné des enfants de FrançoisHenri, Jean-Gilles, est mortellement blessé, en 1707, devant Saragosse; le second est tué en duel à dix-sept ans; le troisième meurt au berceau. En deux ans de mariage Jean-Gilles a eu deux enfants: une fille et un fils. Celui-ci meurt accidentellement en 1773 à la tête de son régiment. La branche des Plessis-Bellière disparaît avec lui (9).

Depuis longtemps déjà le modèle du gentilhomme comporte d'autres vertus que la bravoure au combat. Par touches successives on a enrichi le portrait idéal du noble d'épée. Pour Joinville le "prud'homme", c'est-à-dire le chevalier parfait, doit ajouter au courage, justice, loyauté, foi et charité chrétiennes. L'auteur de Jehan de Saintré lui demande d'être à la messe "le plus dévôt", à table "le plus honneste", "en compaignie des seigneurs et des dames, le plus avenant". Il sera aussi le mieux "monté et abillié" (10). Bayard, quant à lui, a cumulé les plus hautes qualités morales. Le Loyal Serviteur assure qu'

"il aymoit et craignoit Dieu sur toutes choses, ne jamais ne le juroit ne blasphemoit... Il aymoit son prochain comme soy-mesme". "Furieux aux ennemys, (il était) "doulx, paisible, courtois aux amys". "Fort mauvais flateur et adulateur", mais "grand aumosnier", il faisait ses aumones secrètement. "Des biens mondains, il n'y pensa en sa vie" (11).

En somme une sorte de saint que l'Eglise aurait pu canoniser. Mais en général elle n'a pas canonisé les chevaliers, et peut-être avait-elle de bonnes raisons pour ne pas le faire.

Bayard est courageux, fidèle et généreux, mais un peu rustre. Or voici que la Renaissance présente au gentilhomme, du moins s'il fréquente les cours, de nouvelles exigences: à la bravoure il doit ajouter

(9). - Fr. LEBRUN, Les hommes et la mort en Anjou aux XVIIe ct XVIIIe siècles, Paris-La Haye, 1971, pp. 426-427.

(10). - A. DE LA SALE, Jehan de Saintré, p. 30.

(11). - La Tresjoyeuse ... hystoire du bon chevalier ... 2, . 134. 
la culture. Dans le Courtisan publié en 1528 à la demande de François 1er, B. Castiglione, après avoir affirmé que

"la vraie et principale profession du courtisan doit être celle des armes", ajoute: "Je blâme les Français de ce qu'ils disent que les lettres portent nuisance à la profession des armes, estimant n'être à nul plus convenable d'avoir la science des lettres qu'à un homme de guerre ... Sa voix doit être bonne, et non trop déliée ou molle, semblable à celle d'une femme, ni aussi tant austère ni âpre qu'elle tienne du paysan, mais sonnante, claire, douce et bien composée, avec la prononciation franche et nette, contenance et gestes convenables ... Mais toutes ces choses ne serviraient de rien si les sentences et paroles exprimées n’étaient belles, ingénieuses, subtiles, élégantes et graves selon la nécessité” (12).

Héros dans les combats, mais homme de salon lorsqu'il est au repos, le courtisan doit donc avoir fréquenté l'école et les bons auteurs, être tout ensemble César et Aristote, pour reprendre un mot d'Henri II. Il reste que pour Castiglione la première caractéristique du noble, c'est le courage physique, le refus de la peur.

Le défi à la mort, jamais peut-être les gentilshommes ne l'ont lancé avec autant de désinvolture qu'au temps de la Renaissance un défi lié à'l'exaltation de l'honneur. Ce terme revient constamment dans les nombreux écrits du XVIe siècle qui nous livrent les réflexions de la noblesse sur elle-même et sur sa place dans la société: L'Ordre de chevalerie de S. Champier (1510), Les Dialogues d'honneur de G. B. Possevino (trad. fr. 1557), Le Traité des nobles et des vertus dont ils sont formés de François de l'Alouëte (1577), Le Hérault de la noblesse de France de Pierre d'Origny (1578), La Précédence de la noblesse de Guillaume d'Oncien (1593) etc. A l'origine, "honneur" avait désigné une fonction, un fief notamment avec les obligations et dignités qui y étaient attachées. Au temps de la Renaissance, le terme oscille, comme l'a montré Arlette Jouanna, entre quatre significations non exclusives les unes des autres: le mérite qui rend estimable; la réputation, c'est-à-dire l'effet produit sur autrui par le spectacle de qualités ou d'actes conformes à un modèle socialement reconnu; les signes extérieurs qui accompagnent l'estime (on parle alors surtout des "honneurs"); enfin le prestige qui élève au-dessus de "l'horizon commun" (13). De ces quatre sens c'est le second qui prend, au

(12). - Cf. B. CASTIGlione, Il Cortigiano, éd. B. Maier, Turin, $1955, \mathrm{I}, 17$ et 42 , pp. 109 et $157-158$. Ici, trad. fr. du XVIe siècle.

(13). - A. JOUANNA, La notion d'honneur au XVIe siècle, dans Revue d'Histoire Moderne et Contemporaine, oct.-déc. 1968, pp. 597-623. 
XVIe siècle, le pas sur les autres dans le monde des gentilshommes (14). C'est en effet l'époque où triomphe le "point d'honneur". Ce souci de la réputation devient une véritable "loi de classe" à laquelle on ne saurait désobéir.

Il faut souligner l'aspect "externe" de cet honneur chevaleresque indissociable d'une relation entre regardé et regardant. Chamfort dira plus tard:

"L'homme vit souvent avec Jui-même et il a besoin de vertu; il vit avec les autres et il a besoin d'honneur":

formule particulièrement applicable au noble d'épée de la Renaissance auquel incombent deux devoirs: acquérir par des prouesses un surcroît de renommée - c'est l'idéal de Don Quichotte; et ne pas tolérer les "paroles picquantes" qui ternissent une réputation. François $1 \mathrm{er}$, qui s'y entendait en chevalerie, déclara:

"Celui qui endure paroles injurieuses et démenties n'est nullement courageux, ny ne peut estre noble".

Seul le sang de l'insultant peut laver l'honneur de l'insulté qui doit risquer sa vie pour rétablir sa renommée. Un chirurgien peut apporter remède à une blessure. Mais, affirme le sire de la Colombières,

"le gentilhomme blessé d'un trait de langue venimeuse n'a médecin qui le puisse guérir".

Singulière puissance de la parole! Malgré l'idéal chevaleresque d'un François 1er, les rois de France auraient voulu éviter la multiplication des duels. Or ceux-ci, rares aux XIVe et XVe siècles, devinrent ensuite, pendant cent cinquante ans le sport favori de la noblesse d'épée, en dépit des ordonnances de Charles IX, d'Henri IV, et de Louis XIII.

"Il faut, écrivait Brantôme dans son Traité des duels, préférer l'honneur au Prince, à son mandement, à la vie et à tout".

(14). - H. MOREL, La fin du duel judiciaire en France et la naissance du point d'honneur, dans Revue Historique de Droit Français et Etranger, janvier-mars 1964, pp. 574-639. Cf. aussi L. E. HALKIN, Pour une histoire de l'honneur, dans Annales E. S. C., 1949, pp. 443-444. 
Le point d'honneur triompha donc pendant un siècle et demi de l'autorité royale. Durant neuf années du règne d'Henri IV quatre mille gentilshommes français auraient péri en duel. Tel jeune seigneur, le chevalier d'Andrieux, se vantait à trente ans, d'avoir déjà tué soixante douze personnes. Il était donc devenu de bon ton de risquer sa vie à tout propos pour une parole mal sonnante. Bien mieux, l'honneur consistait à provoquer constamment d'autres gentilshommes. A un jeune noble qui lui demandait la main de sa nièce, M. d'Isancourt répondit - on était à l'époque de Richelieu -:

"Il n'est pas encore temps de vous marier. Si vous voulez devenir un honnête homme, il vous faut d'abord tuer en combat singulier deux ou trois hommes, puis vous vous marierez et vous aurez deux ou trois enfants. C'est ainsi que par vous le monde n'aura rien gagné ni perdu" (15).

Le terme "point d'honneur" est d'origine espagnole (pundonor). Pourtant la fureur du duel est venue d'Italie, terre d'élection de la vendetta et des "doctrines duellistes" qui codifièrent les règles de l'honneur. Les guerres d'Italie ne constituèrent toutefois qu'un facteur externe qui cristallisa une évolution. Cet incessant défi à la mort, explique $\mathrm{H}$. Morel, eut des raisons plus profondes et résulta d'une frustration. La multiplication des armées permanentes, le rôle croissant de l'infanterie et des armes à feu avaient diminué sur le champ de bataille le rôle du gentilhomme. Pour tenter de conserver ou de recouvrer une situation sociale compromise, la noblesse exploita à fond le trésor qu'elle possédait "au plus profond de son être": l'honneur", divinité à laquelle elle se sacrifia avec acharnement et désinvolture.

\section{2. - LA CRAINTE "ROTURIERE".}

L'archétype du chevalier sans peur, sinon toujours sans reproche, était rehaussé par le contraste avec une masse réputée sans courage et avec laquelle le gentilhomme ne voulait rien avoir de commun. Au siège de Padoue (en 1509), Bayard s'insurge contre l'avis de l'Empereur Maximilien qui voudrait mettre la gendarmerie française à pied et la faire charger aux côtés des lansquenets:

(15). - G. d'AVENEL, Richelicu et la monarchie absolue, Paris, 1884$-1890,4$ vol., II, p. 80 . 
"l'Empereur pense-t-il que ce soit chose raisonnable de mettre tant de noblesse en péril et hasard avec des piétons dont l'un est cordonnier, l'autre maréchal. l'autre boulanger, et gens mécaniques qui n'ont leur honneur en si grosse recommandation que des gentilshommes?" (16).

Une éthique chevaleresque que double le mépris de la piétaille explique l'hostilité de la noblesse à l'égard des armes à feu.

"Que plust à Dieu, s'exclame Montluc, que ce malheureux instrument (l'arquebuse) n'eust jamais été inventé!".

Et Arioste d'expliquer (en s'adressant, cette fois, au canon, mais l'arrière-plan du raisonnement est le même):

"La gloire militaire est détruite par toi. Par toi, le métier des armes a perdu son honneur. Par toi, valeur et courage sont abolis".

Artillerie et arquebuse permettent au lâche de tirer de loin, sans risquer sa vie. On peut avoir peur et obtenir la victoire. L'équation - simple soldat égale homme sans courage - est toutefois posée par un esprit aussi lucide que Commynes vis-à-vis de la noblesse. In reconnaît que les archers sont devenus

"Ia souveraine chose du monde pour les batailles",

mais à certaines conditions: les réunir par milliers, car en petit nombre ils ne servent à rien; les choisir, si la guerre ne doit pas durer trop longtemps, parmi les soldats qui ne connaissent pas encore la réalité du champ de bataille; les rassurer par la présence auprès d'eux d'une

"grant quantité de nobles et de chevaliers";

leur donner du vin avant le combat afin de stimuler leur ardeur (17).

(16). - La Tresjoyeuse ... hystoire du bon chevalier, 1, p. 307. Cf. G. PROCACCI, Lotta di classe in Francia sotto l'Ancien Régime (1484-1559), dans Societd, sept. 1951, pp. 416-443; F. BRAUDEL, La Méditerranée et le monde méditerranéen d̀ l'époque de Philippe II, 2e éd., Paris, 1966, II, p. 79 .

(17). - COMMYNES, Mémoires, éd. Calmette, Paris, 1924-1925, I, pp. 23-26. Cf. J. DUFOURNET, La destruction des mythes dans les Mémoires de Commynes, Genève, 1966, p. 614. 
Au cours des voyages de découvertes de la Renaissance, les chefs ne sont pas toujours des nobles. Toutefois, maîtres après Dieu sur leur navire, ils entrent moralement dans la catégorie sociale supérieure. Leur courage individuel s'appose alors aux peurs collectives et les narrateurs de l'aventure maritime retrouvent à leur propos le schéma bipolaire familier à ceux qui racontent des batailles ou dissertent sur la noblesse. Zurara dans sa Chronique des faits de Guinée (1453) rehausse les exploits des paladins portugais en insistant sur les "basses frayeurs" des équipages (18). Lors du premier voyage de Christophe Colomb, dès que l'aiguille aimantée dévie quelque peu, les marins "prennent peur" et "demeurent tout confus" (19). Généralisant, Ferdinand Colomb ne voit dans les marins de son père que "des gens portés à la frayeur et aux plaintes". Leur "effroi" quasi permanent est une gêne constante pour le capitaine (20). Faisant rapport à François $1 \mathrm{er}$ en 1524 sur son voyage en Amérique, Verrazzano parle de ses matelots "épouvantés" devant l'attitude de certains Indiens,

"ainsi qu'il advient chaque fois qu'ils se trouvent en presence d'une nouveauté".

L'établissement de Villegaignon au Brésil s'étant heurté dès le début à d'inévitables obstacles, peur et découragement s'emparèrent des hommes et Anon, le chroniqueur de l'expédition, de préciser:

"Les artisans, comme j'ai prédit, gens de petite considération et peu ou point touchés d'honneur, se persuadèrent que la fin serait fort dangereuse, puisque le commencement était tel" (21).

Il arrive à de bons connaisseurs des choses de la guerre de nuancer l'opposition entre le courage du chef et la lâcheté du vulgaire. Significatif à cet égard cet éloge des fantassins par Brantôme - lequel n'a pas toujours été un écrivain "frivole":

“... Je pense qu'il n'y a rien si brave et si superbe à voir qu'un gentil soldat bien en poinct, bien armé, bien leste, soit

\footnotetext{
(20). - J. CASSOU, Les découvertes du Nouveau Monde, Paris, 1966, p. 205

(21). - ANON, Histoire des choses mémorables advenues en la Terre du Brésil, 1561, 10vo.
} p. 35 .

(18). - Trad. fr. de L. BOURDON, Dakar, 1960.

(19). - Ch. ColomB, Oeuvres, trad. A. Cionarescu, Paris, 1961, 
qu'il marche à la teste d'une compaignie, soit qu'il se perde devant tous à un'escarmouche, ou à un assaut tirer son harquebuzade tout nud, desarmé, aussi résolument que les mieux armez... Et ce que j'admire en ces fantassins, c'est que vous verrez de jeunes gens sortir des village, de la labeur, des boutiques, des escoles, des pallais, des postes, des forges, des escuries, des lacquays et de plusieurs autres lieux pareils, bas et petitz; ils n'ont pas plus tost demeurez parmi cette infanteric quelque temps, que vous les voyez aussitost faitz, aguerrys, façonnez, que, de rien qu'ilz estoient, viennent à être capitaines et esgaux aux gentilz-hommes, ayant leur honneur en recommandation autant que les plus nobles, à faire actes auusi vertueux et nobles que les plus grands gentilz-hommes. Voyez quelle obligation ilz ont aux armes qui les poussent ainsi!" (22).

Au vrai, Brantôme n'apporte ici qu'une variante au schéma hiérarchique d'une société d'ordres qui contraste la vaillance du chevalier par la pusillanimité du vilain. Par le métier militaire, des paysans, des artisans, des valets accèdent pratiquement à la noblesse. Aussi bien acquièrent-ils la notion d'honneur et recherchent-ils les prouesses individuelles; Brantôme est donc logique avec lui-même lorsqu'il soutient, contre La Noue, que les soldats ne doivent plus ensuite retourner à leurs activités premières. Car il ne faut pas que les mains qui ont manié les armes

"si noblement et si nettement s'aillent souiller et vilanner par un labourage vil et salle mestier mécanique".

Mieux vaut repartir mercenaire, en Turquie ou en Barbarie (23). On en revient donc bien à l'opposition fondamentale entre une minorité courageuse parce que guerrière, et une masse qui n'a que faire de la vaillance ni d'autre but dans l'existence que de survivre.

Cette incompatibilité entre deux univers à la fois sociaux et moraux - celui de la témérité et celui de la peur - le roman et le thêâtre ne manquent pas de la souligner, même si le gentilhomme mis en scène est fantasque comme Don Quichotte ou odieux comme Don Juan. Don Quichotte se préparant à intervenir pour l'armée de Pentapolin contre celle du "grand empereur Alifanfaron" - un "furieux païen" - Sancho Pança fait timidement remarquer à son maître qu'il

(22). - BRANTOME, Discours sur les colonels de l'infanterie de France, éd. E. Vaucheret, Montréal-Paris, 1973, p. 98.

(23). - Ibid., p. 105. 
s'agit simplement de deux troupeaux de moutons. La réponse du chevalier est conforme à la représentation mentale que, des siècles durant, la noblesse d'épée a eue d'elle-même et de la masse des vilains:

"C'est la peur que tu as qui te fait, Sancho, voir et entendre tout de travers; car l'un des effets de cette triste passion est de troubler les sens, et de faire paraitre les choses autrement qu'elles ne sont. Mais, si ta frayeur est si grande, retire-toi à l'écart et laisse-moi seul; seul, je donnera la victoire au parti où je porterai le secours de mon bras" (24).

Prouesses individuelles toujours, mais sacrilèges cette fois, de Don Juan, "l'abuseur de Séville", qui défie le spectre du commandeur, Dieu et l'enfer. Naturellement, son serviteur Catherinon va de frayeur en frayeur et Don Juan le lui reproche:

"Quelle peur as-tu d'un mort? Que ferais-tu si c'était un vivant? Sotte et roturière crainte!" (25).

L'imagination chevaleresque de Don Quichotte transforme moutons et moulins à vent en ennemis. Toutefois la peur est, plus souvent que la vaillance, à l'origine de telles illusions. A preuve ce texte de Montaigne dans lequel la propension à la frayeur est tout naturellement attribuée aux humbles, même lorsqu'ils sont soldats:

"De vrai, j'ai vu beaucoup de gens devenus insensés de peur... Je laisse à part le vulgaire à qui elle représente tantôt les bisaïeux sortis du tombeau, enveloppés en leur suaire, tantôt des loups-garous, des lutins et des chimères. Mais parmi les soldats même, où elle devrait trouver moins de place, combien de fois a-t-elle changé un troupeau de brebis en escadron de corselets (cuirassiers)? Des roseaux et des canes en gens d'armes et lanciers? Nos amis en nos ennemis? Et la croix blanche à la rouge?" (26).

Montagne associe ailleurs lâcheté et cruauté, l'une et l'autre étant plus spécialement le fait de "cette canaille de vulgaire":

(24) . CERVANTES, Don Quichotte, Trad. L. Viardot, Paris, s. d., I. ch. XVIII, p. 126.

(25). - TIRSO DE MOLINA, L'abuseur de Séville. Don Juan, Acte III. Trad. P. Guenoun, Paris, 1968, p. 159.

(26). - MONTAIGNE, Essais, I, ch. XVIII (De la peur), éd. A. Thibaudet, Paris, 1965, p. 106. 
"Les meurtres des victoires s'exercent ordinairement par le peuple et par les officiers du bagage; et ce qui fait voir tant de cruautés inouïes aux guerres populaires, c'est que cette canaille de vulgaire s'aguerrit et se gendarme à s'englanter jusques aux coudes et à déchiqueter un corps à ses pieds, n'ayant ressentiment (sentiment) d'autre vaillance..." (27).

Couardise et cruauté sont souvent liées, et Montaigne a raison de le souligner. Mais pourquoi les roturiers se priveraient-ils du droit légitime d'avoir peur au moment du danger? Ils n'ont pas "d'honneur" à préserver, du moins dans le sens où la noblesse d'épée entend ce terme. Ils n'accèderont pas à la gloire. Ils sont voués toute leur vie à l'anonymat. Dès lors, à quoi bon prouesses et témérité? L'idée que la masse des paysans, des artisans et des serviteurs n'est pas courageuse parce qu'elle ne recherche pas - et ne peut pas rechercher la renommée a eu cours longtemps et se trouve encore à la fin du XVlle siècle sous la plume d'un La Bruyère, par ailleurs sans indulgence particulière pour les gentilshommes:

"s'il est vrai qu'un grand donne plus à la Fortune lorsqu'il hasarde une vie destinée à couler dans les ris, le plaisir et l'abondance, qu'un particulier qui ne risque que des jours qui sont misérables, il faut avouer aussi qu'il y a un tout autre dédommagement, qui est la gloire et la haute réputation. Le soldat ne sent pas qu'il soit connu; il meurt obscur et dans la foule: il vivait de même, à la vérité, mais il vivait; et c'est l'une des sources du défaut de courage dans les conditions basses et serviles. Ceux au contraire que la naissance démêle d'avec le peuple et expose aux yeux des hommes, à leur censure et à leurs éloges, sont même capables de sortir par effort de leur tempérament, s'il ne les portait pas à la vertu; et cette disposition de coeur et d'esprit, qui passe des aïeuls par les pères dans leurs descendants, est cette bravoure si familière aux personnes nobles, et peut-être la noblesse même. Jetez-moi dans les troupes comme un simple soldat, je suis Thersite (28); mettez-moi à la tête d'une armée dont j’aie à répondre à toute l'Europe, je suis Achille" (29).

Ce lieu commun - la masse est peureuse - doit être encore précisé par deux notations, contradictoires dans leurs motivations mais

(27). - Ibid., II, ch. XXVII (Couardise mère de cruauté), éd. P. Michel, Paris, 1965, p. 357.

(28). - Dans PIliade, Thersite personnifie la couardise.

(29). - LA BRUYERE, Les Caractères (Des Grands, 41), éd. R. Garopon, Paris, 1962, Pp. 266-267. 
convergentes par l'éclairage qu'elles apportent et qu'on peut ainsi résumer: la classe dirigeante a fait en sorte que le peuple - essentiellement les paysans - ait peur. Symphorien Champier, médecin et humaniste mais thruriféraire de la noblesse, écrit en effet: le seigneur doit prendre

"aise et délit des choses dont ses hommes ont paine et travail". Son rôle est de "maintenir terre car pour la paour que les gens du peuple ont des chevaliers ilz labourent et cultivent les terres par paour et crainte d'estre destruictz" (30).

Quant à Thomas More, qui conteste la société de son temps en se situant toutefois prudemment dans une imaginaire "Utopie", il affirme que

"La pauvreté du peuple est la défense de la monarchie... L'indigence et la misère enlèvent tout courage, abrutissent les âmes, les accommodent à la souffrance et à l'esclavage et les oppriment au point de leur ôter toute énergie pour secouer le joug" (31).

Résumons: la peur des uns est la condition du courage des autres; et la lâcheté - collective - des peuples dont le destin est d'être soumis révèle par contraste la vaillance - individuelle - des héros qui dirigent la société.

JEAN DELUMEAU. Né à Nantes le 18 juin 1923.

Entré à l'École Normale Supérieure (Lettres) en 1943.

Engagé volontaire en décembre 1944 et militaire jusqu'en janvier 1946.

Agrégé d'histoire en 1947.

Professeur au Lycée Alain-Fournier de Bourges en 1947. -1948 .

(30). - L'Ordre de chevalerie (1510) traité publié dans P. ALLUT, Etude historique et bibliographique sur $S$. Champier, Lyon, 1899, pp. 75-76.

(31). - Th. MORE, L'Utopie, éd. V. Stouvenel, Paris, 1945, p. 75. 
Membre de l'École Française de Rome d'octobre 1948 a octobre 1950.

Professeur de Première Supérieure au Lycée Chateaubriand de Rennes d'octobre 1950 à octobre 1954.

Détaché au C. N. R. S. d'octobre 1954 a octobre 1955.

Docteur ès-Lettres (Histoire) en juin 1955.

Maitre de Conférences, puis professeur d'Histoire Moderne à Rennes d'octobre 1955 à octobre 1970.

Professeur d'Histoire Moderne à Paris I d'octobre 1970 à octobre 1974.

Directeur d'Études a l'École Pratique des Hautes Etudes (VIe Section) depuis octobre 1963.

Directeur du Centre Armoricain de Recherches Historiques de 1964 a 1970.

Directeur du Centre d'Histoire Moderne de Paris I de 1970 à 1974.

Professeur au Collège de France depuis octobre 1974.

Membre de la Société des Italianistes de l'Enseignement Supérieur.

Médaille d'Argent du C. N. R. S. 1962.

Officier des Palmes Académiques.

Chevalier de l'Ordre du Mérite.

\section{Bibliographie (ouvrages).}

Vie économique et sociale de Rome dans la seconde moitié du XVIe siècle (thèse de doctorat d'Etat), 2 vol.,. Paris, De Boccard, 1957-1959. $1.038 \mathrm{p}$. Bibliothèque des Ecoles Françaises d'Athènes et de Rome.

L'Alun de Rome (thèse complémentaire). Paris. S. E. V. P. E. N. 1962. $352 \mathrm{p}$.

Naissance et affirmation de la Réforme. Paris. P. U. F., coll:

"Nouvelle Clio". 1965, 417 p.; 2éd. 1968; 3e éd. 1973.

La Civilisation de la Renaissance. Paris. Arthaud. coll. "Les Grandes Civilisations". 1967, 717 p.

Histoire de la Bretagne (direction de l'). Toulouse. Privat. Coll. "L'Univers de la France". 1969. 542 pp.

Documents de Phistoire de la Bretagne. Toulouse. Privat. 1971. 402 p.

Le Catholicisme entre Luther et Voltaire. Paris. P. U. F. Coll. "Nouvelle Clio". 1971. 358 p.

L'Italie de Botticelli à Bonaparte. Paris. A. Colin. 1974. 340 p. 